\title{
Multicentric Reticulohistiocytosis Associated with Liver Carcinoma: Report of a Case
}

\author{
Ling Han Qiong Huang Kang-Huang Liao Lian-Jun Chen \\ Wen-Yi Kong Wen-Wen Fu Jing-hua Xu \\ Department of Dermatology, Huashan Hospital, Fudan University, Shanghai, PR China
}

\section{Key Words}

Multicentric reticulohistiocytosis $\cdot$ Liver carcinoma $\cdot$ Malignancy

\begin{abstract}
We report a unique case of multicentric reticulohistiocytosis (MRH) associated with liver carcinoma. A 61-year-old man presented with a 4-month history of nonpruritic, generalized, ruby-red papules and nodules, accompanied by fever, joint swelling and difficulty in swallowing. Skin histology showed polymorphic histiocyte infiltration with typical 'ground glass' cytoplasm. Further immunohistochemical studies characterized the lesions as positive for leukocyte common antigen, HLA-DR and CD68. The patient had a history of hepatitis B, and systemic examination, including carcinoma index and type-B ultrasonic examination, revealed high levels of AFP and a solid tumor, which was considered malignant, localized on the right lobe of the liver. Treatment of the liver carcinoma resulted in a significant improvement of the skin symptoms. This is the first case study to report an association between MRH and liver carcinoma. A review of the English-language literature reveals the close linkage between MRH and malignancy. All patients with MRH should be evaluated and monitored carefully to determine the underlying neoplasm.
\end{abstract}

\section{Introduction}

Multicentric reticulohistiocytosis (MRH) is a rare multisystem syndrome characterized by polyarthritis and papulonodular skin lesions with typical dermal infiltration of histiocytes and multinucleated giant cells. The disease may involve the skin, tendon sheath, synovium, bone, liver, salivary gland, kidney, lymph node, heart and lung. In MRH, an association with hyperlipidemia (30-58\%), a positive skin tuberculin test (12-50\%), systemic vasculitis and autoimmune disease has been described [1]. 
Of utmost clinical importance is the association of MRH with a variety of underlying internal malignancies. Such an association has been documented in up to $28 \%$ of all reported cases in the world literature, the most common being bronchial, breast, stomach, and cervical carcinomas [2]. Here, we report, for the first time, a case of MRH presenting with liver carcinoma, thus highlighting the association of MRH with malignant disease.

\section{Case Report}

A 61-year-old man presented with a 4-month history of a nonpruritic rash, which started on his forearm and face after sun exposure and subsequently spread to the ear, scalp and upper chest, after which asymptomatic skin lesions appeared on the lateral margin of his finger. The patient denied any history of photosensitivity but complained of low-grade fever and painful swelling of the joints in his wrist, knee and ankle, accompanied by restricted activity for 1 month. He had a sensation of a foreign body in his pharynx with difficulty in swallowing for about 1 month.

The patient also had a history of hepatitis B, which was diagnosed in the 1970's, and severe knee joint pain with bony spurs, which were confirmed by X-ray, for at least 2 years. There was no history of drug allergy, operation, trauma or inherited disease. Treatment with hydroxychloroquine and thalidomide for solar dermatitis in other hospitals showed no therapeutic effect.

Physical examination showed a confluent erythematous eruption affecting his scalp, face, neck and the extensor aspect of his forearm, which was characterized by ruby-red, translucent and grouped papules (fig. 1a) that were $0.5 \mathrm{~mm}$ to $1 \mathrm{~cm}$ in diameter. Red nodules could also be found on the dorsum of the nose, lateral area of the fingers and tongue tip (fig. 1b). Musculoskeletal examination revealed a painful swelling of the wrist and knee joints. The results of the clinical examination were otherwise unremarkable.

An extensive laboratory evaluation, including white cell count, urinalysis, stool studies, renal function, electrolytes, cholesterol and triglycerides, erythrocyte sedimentation rate, antistreptolysin 0 level, rheumatoid factor and antinuclear antibody were normal. Liver function tests including AST 34 U/l (<30 U/l), ALT 125 U/l (<50 U/l), ALP 159 U/l (35-104 U/l), GGT 100 U/l (3-45 U/l), total bilirubin $30 \mu \mathrm{mol} / \mathrm{l}(3.4-20.4 \mu \mathrm{mol} / \mathrm{l}), \mathrm{TBA} 21 \mu \mathrm{mol} / \mathrm{l}(<6.8 \mu \mathrm{mol} / \mathrm{l})$ and total protein, albumin and globulin were normal. Hepatitis index studies showed $\mathrm{HBsAg}(+), \mathrm{HBsAb}<0.2 \mathrm{IU} / \mathrm{l}(>10 \mathrm{IU} / \mathrm{l})$, $\operatorname{HBeAb}(+), \operatorname{HBsAb}<\operatorname{HBeAg}(-), \operatorname{HBeAb}(+), \operatorname{HBcAb}(+), \operatorname{HBcIgM}(-), \operatorname{HAVIgM}(-)$ and $\operatorname{HCV}(-)$. The AFP level was significantly elevated $(6,820 \mu \mathrm{g} / \mathrm{l}$, normal range $<10 \mu \mathrm{g} / \mathrm{l})$. Type-B ultrasonic examination revealed a solid tumor $(91 \times 69 \mathrm{~mm})$ on the right lobe of the liver, which was suspected to be a small hepatocellular carcinoma.

Treatment with hydroxychloroquine and thalidomide were ineffective. The patient then received hepatic arterial chemoembolization followed by percutaneous transhepatic cholangiography drainage. Five-FU, Oxaliplatin and THP were used as anticancer drugs. The AFP level after the interventional treatment was $1,027.5 \mu \mathrm{g} / \mathrm{l}$, and the tumor mass decreased to $67 \times 66 \mathrm{~mm}$. The cutaneous symptoms improved significantly within 1 month (several large nodules disappeared immediately; later, the erythematous rash and grouped papules resolved and the patient's arthralgia and swelling of joints gradually improved).

Skin biopsy on the right forearm showed infiltration of the dermis with polymorphic histiocytes and giant cells with 'ground glass' cytoplasm lying between collagen bundles (fig. 2a). The histiocytic marker CD68 was positive, as were leukocyte common antigen, HLA-DR, lysozyme and vimentin (fig. $2 \mathrm{~b}, \mathrm{c}$ ). Langerhans cell markers (S-100 and CD1a) were negative and the histological appearances were consistent with MRH. 


\section{Discussion}

$\mathrm{MRH}$, often referred to as lipoid dermato-arthritis, was first reported in 1937 by Weber and Freudenthal [3]. MRH is so rare that fewer than 300 cases have been reported in the literature. In 1969, Barrow and Holubar [4] first raised the question of whether there was an association between MRH and malignancy. They found 5 cases of cancer in 33 patients (15\%). Snow and Muller [5] reported a malignancy rate of $25 \%$ in 133 cases of MRH reported in the literature until 1994. The malignancies were most commonly hematological, breast or stomach carcinomas.

Eleven [6-15] of the 66 cases we reviewed (English-language literature from 1995 until now) had cancer and were evaluated for the following parameters: age, sex, type of neoplasm at presentation, duration preceding or following the diagnosis of cancer that MRH developed and treatment response of both the tumor and MRH. The results are presented in table 1 (including the present case). For most of these cases (including the present case), the onset of malignancy and MRH occurred within approximately 3 years, and in some cases, the onset of MRH also occurred in close proximity to the time of recurrence of a previously diagnosed malignancy $[13,15]$.

This is the first case report of MRH with liver carcinoma. Although the etiology of MRH is unknown, the possibility that MRH reflects a constellation of symptoms seen within paraneoplastic syndrome is supported by the frequency of associated neoplasm (18\% based on our review), the remission of the lesions after tumor treatment ( 6 of 12 cases [6-8, 10-12]) and the onset of MRH just prior to the relapse of the neoplasm (case No. 8 [11] of table 1). Therefore, all patients with MRH should be evaluated and monitored carefully. 
Table 1. Review of cases of malignancy-associated MRH in the English-language literature

\begin{tabular}{|c|c|c|c|c|c|c|c|c|}
\hline \multirow[t]{2}{*}{ Case No. } & \multirow{2}{*}{$\begin{array}{l}\text { Sex/age } \\
\text { years }\end{array}$} & \multirow{2}{*}{$\begin{array}{l}\text { Type of } \\
\text { malignancy }\end{array}$} & \multirow{2}{*}{$\begin{array}{l}\text { Time of } \\
\text { malignancy } \\
\text { relative to } \\
\text { MRH }^{\mathrm{a}}\end{array}$} & \multirow[t]{2}{*}{ Treatment } & \multicolumn{3}{|c|}{ Response to treatment } & \multirow[t]{2}{*}{ Outcome } \\
\hline & & & & & tumor & $\begin{array}{l}\text { skin } \\
\text { mani- } \\
\text { festation }\end{array}$ & $\begin{array}{l}\text { MRH } \\
\text { arthritis }\end{array}$ & \\
\hline $\begin{array}{l}1 \\
\text { (present } \\
\text { case) }\end{array}$ & M/61 & $\begin{array}{l}\text { liver } \\
\text { cancer }\end{array}$ & +4 months & $\begin{array}{l}\text { (1) immunosuppressive } \\
\text { agent } \\
\text { (2) hepatic arterial } \\
\text { chemoembolizaion } \\
\text { (3) PCTD external } \\
\text { drainage }\end{array}$ & $\begin{array}{l}- \\
\text { PR } \\
\text { PR }\end{array}$ & $\begin{array}{l}\text { NSC } \\
\text { GI } \\
\text { GI }\end{array}$ & $\begin{array}{l}\text { GI } \\
\text { GI }\end{array}$ & $\begin{array}{l}\text { MRH: } \\
\text { improved } \\
\text { malignancy: } \\
\text { PR }\end{array}$ \\
\hline $2[6]$ & $\mathrm{F} / 62$ & $\begin{array}{l}\text { lung } \\
\text { cancer }\end{array}$ & +1 year & $\begin{array}{l}\text { (1) steroids } \\
\text { (2) MTX } \\
\text { (3) right lower lobectomy }\end{array}$ & $\begin{array}{l}- \\
\overline{C R}\end{array}$ & $\begin{array}{l}\mathrm{CR} \\
\mathrm{CR} \\
\text { no recurrence }\end{array}$ & $\begin{array}{r}P R \\
P R \\
\text { ePR }\end{array}$ & $\begin{array}{l}\text { no recurrence } \\
\text { of MRH and } \\
\text { cancer }\end{array}$ \\
\hline $3[7]$ & $\mathrm{F} / 56$ & $\begin{array}{l}\text { urinary bladder } \\
\text { cancer }\end{array}$ & $S$ & $\begin{array}{l}\text { (1) steroids } \\
\text { (2) cisplatin } \\
\text { (3) etoposide }\end{array}$ & $\begin{array}{l}- \\
\text { PR } \\
\text { PR }\end{array}$ & $\begin{array}{l}\text { NSC } \\
\text { PR } \\
\text { PR }\end{array}$ & $\begin{array}{l}\text { PR } \\
- \\
-\end{array}$ & $\begin{array}{l}\text { patient died } 1 \\
\text { month later } \\
\text { from } \\
\text { metastases }\end{array}$ \\
\hline $4[7]$ & $\mathrm{M} / 59$ & renal cancer & $S$ & NM & NM & NM & NM & - \\
\hline $5[8]$ & $\mathrm{F} / 33$ & $\begin{array}{l}\text { nasopharyngeal } \\
\text { caner }\end{array}$ & S & $\begin{array}{l}\text { (1) radiotherapy } \\
\text { (2) cisplatin }\end{array}$ & $\begin{array}{l}\text { PR } \\
\text { PR }\end{array}$ & $\begin{array}{l}\text { PR } \\
\text { PR }\end{array}$ & - & $\begin{array}{l}\text { MRH: } \\
\text { improved } \\
\text { malignancy: } \\
\text { NK }\end{array}$ \\
\hline 6 [9] & $\mathrm{M} / 31$ & $\begin{array}{l}\text { Burkitt } \\
\text { lymphoma and } \\
\text { adenocarcinoma }\end{array}$ & $\begin{array}{l}-24 \text { years } \\
-4 \text { months }\end{array}$ & $\begin{array}{l}\text { (1) CT } \\
\text { (2) radiotherapy } \\
\text { NM }\end{array}$ & $\begin{array}{l}\text { PR } \\
\text { PR } \\
\text { NM }\end{array}$ & $\begin{array}{l}\text { NM } \\
\text { NM } \\
\text { NM }\end{array}$ & $\begin{array}{l}\text { NM } \\
\text { NM } \\
\text { NM }\end{array}$ & - \\
\hline $7[10]$ & $\mathrm{F} / 64$ & $\begin{array}{l}\text { ovarian } \\
\text { cancer }\end{array}$ & +2 months & $\begin{array}{l}\text { (1) steroids } \\
\text { (2) MTX } \\
\text { (3) TH } \\
\text { (4) CT }\end{array}$ & $\begin{array}{l}- \\
- \\
\text { CR } \\
\text { CR }\end{array}$ & $\begin{array}{l}\text { NSC } \\
\text { NSC } \\
\text { CR } \\
\text { CR }\end{array}$ & $\begin{array}{l}\text { GI } \\
\text { GI } \\
\text { CR } \\
\text { CR }\end{array}$ & $\begin{array}{l}\text { MRH: } \\
\text { improved } \\
\text { malignancy: } \\
\text { NR }\end{array}$ \\
\hline $8[11]$ & $\mathrm{F} / 69$ & $\begin{array}{l}\text { endometrial } \\
\text { cancer }\end{array}$ & +3 years & $\begin{array}{l}\text { (1) MTX } \\
\text { (2) TH } \\
\text { (3) CT }\end{array}$ & $\begin{array}{l}- \\
\mathrm{CR} \\
\mathrm{CR}\end{array}$ & $\begin{array}{l}\mathrm{CR} \\
\mathrm{CR} \\
\mathrm{CR}\end{array}$ & $\begin{array}{l}\mathrm{CR} \\
\mathrm{CR} \\
\mathrm{CR}\end{array}$ & $\begin{array}{l}\text { cancer } \\
\text { recurrence } \\
1 \text { year } \\
\text { later/MRH } \\
\text { coalesce }\end{array}$ \\
\hline 9 [12] & $\mathrm{F} / 70$ & $\begin{array}{l}\text { breast } \\
\text { cancer }\end{array}$ & - 15 years & $\begin{array}{l}\text { (1) steroids } \\
\text { (2) MTX } \\
\text { (3) left mastectomy for } \\
\text { breast cancer } 15 \text { years } \\
\text { ago }\end{array}$ & $\begin{array}{l}\text { NK } \\
\text { NK }\end{array}$ & $\begin{array}{l}\mathrm{CR} \\
\mathrm{CR} \\
-\end{array}$ & $\begin{array}{l}P \\
P \\
-\end{array}$ & $\begin{array}{l}\text { no recurrence } \\
\text { of cancer. }\end{array}$ \\
\hline 10 [13] & $\mathrm{F} / 40$ & $\begin{array}{l}\text { breast } \\
\text { cancer }\end{array}$ & $\begin{array}{l}\text { primary: } \\
\text { - } 2 \text { years } \\
\text { recurrence: } \\
+2 \text { months }\end{array}$ & $\begin{array}{l}\text { (1) immunosuppressive } \\
\text { agent } \\
\text { (2) steroids }\end{array}$ & $\begin{array}{l}\text { NK } \\
\text { NK }\end{array}$ & $\begin{array}{l}\text { GI } \\
\text { GI }\end{array}$ & $\begin{array}{l}\text { GI } \\
\text { GI }\end{array}$ & $\begin{array}{l}\text { malignancy: } \\
\text { NR }\end{array}$ \\
\hline $11[14]$ & $\mathrm{F} / 39$ & $\begin{array}{l}\text { unknown } \\
\text { primary tumor }\end{array}$ & S & tamoxifen & PR & NSC & NSC & - \\
\hline 12 [15] & $\mathrm{F} / 65$ & $\begin{array}{l}\text { malignant } \\
\text { melanoma }\end{array}$ & $\begin{array}{l}\text { primary: } \\
\text { - } 9 \text { years } \\
\text { recurrence: } \\
+6 \text { months }\end{array}$ & $\begin{array}{l}\text { (1) steroids } \\
\text { (2) cyclophosphamide } \\
\text { (3) radical dissection }\end{array}$ & $\begin{array}{l}\mathrm{P} \\
\mathrm{P} \\
\mathrm{CR}\end{array}$ & $\begin{array}{l}\text { PR } \\
\text { PR } \\
\text { NK }\end{array}$ & $\begin{array}{l}P \\
P \\
\text { NK }\end{array}$ & $\begin{array}{l}\text { malignancy: } \\
\text { no metastatic } \\
\text { lesions during } \\
5 \text { months }\end{array}$ \\
\hline
\end{tabular}

a Malignancy was detected before (-), after (+), or simultaneously (S) with the onset of MRH. NSC = No significant change; GI = gradually improved; $\mathrm{PR}=$ partial remission; $\mathrm{PCTD}=$ percutaneous transhepatic cholangiography drainage; $\mathrm{CR}=$ complete clinical remission; $\mathrm{NM}=$ no mention; $\mathrm{NK}=$ not known; $\mathrm{CT}$ = chemotherapy; $\mathrm{TH}$ = total hysterectomy; $\mathrm{NR}=$ no remission; $\mathrm{P}=$ progression. 

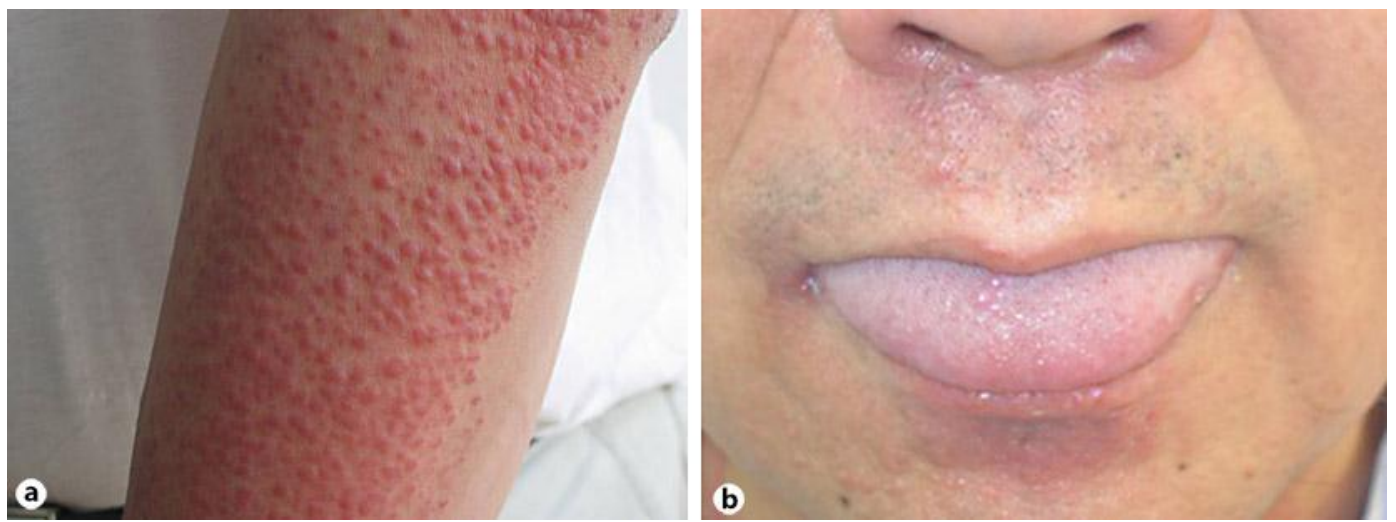

Fig. 1. a Multiple fusion ruby-red papulonodular eruption in the neck, extensor aspect of the forearm and chest (sharply demarcated). $\mathbf{b}$ Light pink and shiny nodule on philtrum and tongue tip. 

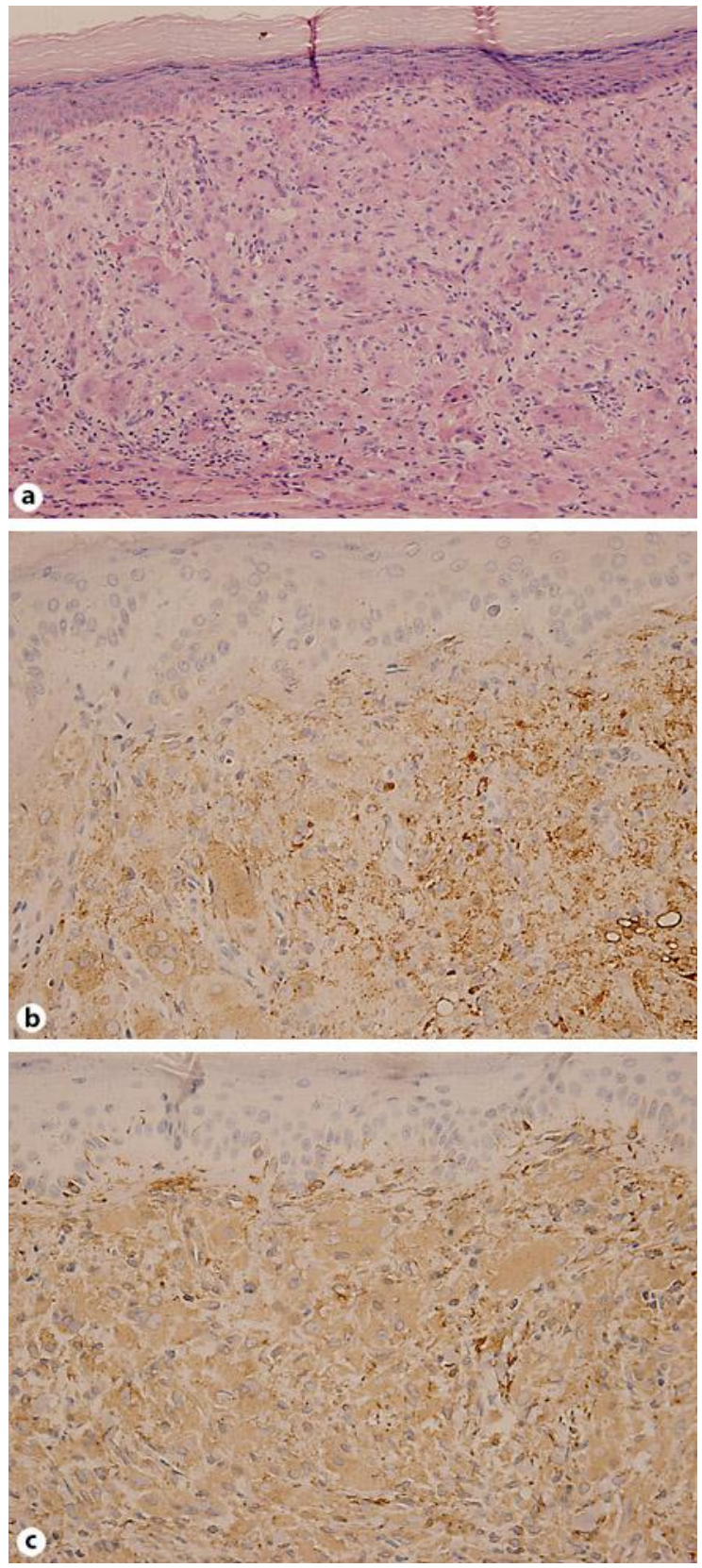

Fig. 2. a H\&E section showing a prominent dermal histocytic infiltrate with typical 'ground glass' cytoplasm $(\times 200)$. Skin samples obtained were studied by immunohistochemical staining. CD68 staining (b) and also Vim positive cells (c) were observed in some histocytes (Envision, $\times 400$ ). 


\section{References}

1 Tajirian AL, Malik MK, Robinson-Bostom L, Lally EV: Multicentric reticulohistiocytosis. Clin Dermatol 2006;24:486-492.

-2 Luz FB, Gaspar TAP, Kalil-Gaspar N, Ramos-e-Silva M: Multicentric reticulohistiocytosis. J Eur Acad Dermatol Venereol 2001;15:524-531.

3 Weber Parkes F, Freudenthal W: Nodular non-diabetic cutaneous xanthomatosis with hypercholesterolemia and atypical histological features. Proc R Soc Med 1937;30:522-526.

4 Barrow MV, Holubar K: Multicentric reticulohistiocytosis. A review of 33 patients. Medicine (Baltimore) 1969;48:287-305.

55 Snow JL, Muller SA: Malignancy-associated multicentric reticulohistiocytosis: a clinical, histological and immunophenotypic study. Br J Dermatol 1995;133:71-76.

-6 El-Haddad B, Hammoud D, Shaver T, Shahouri S: Malignancy-associated multicentric reticulohistiocytosis. Rheumatol Int 2011;31:1235-1238.

7 Tan BH, Barry CI, Wick MR, White KP, Brown JG, Lee A, Litchfield AH, Lener EV, Shitabata PK: Multicentric reticulohistiocytosis and urologic carcinomas: a possible paraneoplastic association. J Cutan Pathol 2011;38:43-48.

8 Teo HY, Goh BK: Multicentric reticulohistiocytosis in association with nasopharyngeal carcinoma. Clin Exp Dermatol 2009;34:e347-e348.

-9 Hinchman KF, Wu JJ, Soden CE Jr, Waldman J, Dyson SW: Multicentric reticulohistiocytosis associated with Burkitt lymphoma and adenocarcinoma. Cutis 2008;82:113-114.

10 Kishikawa T, Miyashita T, Fujiwara E, Shimomura O, Yasuhi I, Niino D, Ito M, Amenomori M, Osumimoto H, Osumi M, Eguchi K, Migita K: Multicentric reticulohistiocytosis associated with ovarian cancer. Mod Rheumatol 2007;17:422-425.

11 Malik MK, Regan L, Robinson-Bostom L, Pan TD, McDonald CJ: Proliferating multicentric reticulohistiocytosis associated with papillary serous carcinoma of the endometrium. J Am Acad Dermatol 2005;53:1075-1079.

12 Santilli D, Lo Monaco A, Cavazzini PL, Trotta F: Multicentric reticulohistiocytosis: a rare cause of erosive arthropathy of the distal interphalangeal finger joints. Ann Rheum Dis 2002;61:485-487.

13 Valencia IC, Colsky A, Berman B: Multicentric reticulohistiocytosis associated with recurrent breast carcinoma. J Am Acad Dermatol 1998;39:864-866.

14 Worm M, Kleine-Tebbe A, von Stebut E, Haas N, Kolde G: Multicentric reticulohistiocytosis indicating metastasis of an unknown primary tumour. Acta Derm Venereol 1998;78:67-68.

15 Gibson G, Cassidy M, O'Connell P, Murphy GM: Multicentric reticulohistiocytosis associated with recurrence of malignant melanoma. J Am Acad Dermatol 1995;32:134-136. 